\section{THU0617 WRIST ULTRASOUND (US) PATHOLOGY IN EARLY RHEUMATOID ARTHRITIS (RA); OBSERVATIONS FROM AN EARLY INFLAMMATORY ARTHRITIS (EIA) DIAGNOSTIC SERVICE}

Rizwan Rajak ${ }^{1}$, Momina Zaman ${ }^{2}$, Tim Jones ${ }^{2}$, Fazal Sheikh ${ }^{3}$, Mohammed Sharif ${ }^{4}$. ${ }^{1}$ Croydon Health Services NHS Trust, Rheumatology, Croydon, United Kingdom; ${ }^{2}$ St George's University Hospital NHS Trust, Rheumatology, Tooting, United Kingdom; ${ }^{3}$ Epsom and St Helier's NHS Trust, Rheumatology, Carshalton, United Kingdom; ${ }^{4}$ King's College Hospital NHS Foundation Trust, Rheumatology, Orpington, United Kingdom

Background: To ensure a timely diagnosis of RA, diagnostic US is increasingly being utilized to detect subclinical pathology in order to implement treatment plans rapidly. There are several 'optimal' synovitis scoring protocols in the literature but a consensus regarding a definitive system still eludes us. There are few studies that have focussed specifically on wrist US pathology in early RA. Hence, we assessed what the common wrist US pathologies are in patients diagnosed with early RA.

Objectives: To identify the patterns of wrist US abnormalities seen in patients diagnosed with early RA from our EIA service in a large urban London hospital.

Methods: Retrospective service review of patients seen in the EIA US diagnostic service at Croydon Health Services in South London. Patients diagnosed with RA (EULAR/ACR 2010 criteria) with wrist US synovitis/ tenosynovitis (EULAR-OMERACT definitions) between Jan2017-Dec2018 were included. The US protocol in the EIA diagnostic service includes lateral and dorsal (long axis \& short axis views) of the ulnarcarpal(UCJ) \& radiocarpal(RCJ) joints with views covering the radioulnar joints \& ulnar-styloids and views of the extensor \& flexor tendons. Images and reports were reviewed and correlations with rheumatoid factor(RhF), antiCCP antibodies(CCP), CRP \& ESR were also assessed.

Results: 86 patients with RA (meeting EULAR-ACR criteria) were found to have wrist pathologies on US. The commonest finding (36\%) was moderate (grade 2) greyscale(GSUS) synovitis in the UCJ with almost all having moderate (grade 2) power Doppler(PDUS) synovitis (32\%). Only a few (5/86) had more severe pathology with GSUS/PDUS (grade 3). $22 \%$ (19/86) had milder changes GSUS grade 1 with three quarters of these patients having concomitant PDUS signal. Just under $10 \%$ had RCJ US synovitis and 3 cases had radioulnar joint synovitis. Only 6\% of the cohort had whole wrist joint involvement. Erosions were very uncommon $(2 / 86)$. In total just under $25 \%(21 / 86)$ had tendon disease with roughly $70 \%$ affecting 1 tendon compartment and the others affecting 2 or more Of those with tendon pathology, the two most commonly affected tendons were the $2^{\text {nd }}[48 \%(10 / 21)]$ and $4^{\text {th }}[38 \%(8 / 21)]$ extensor compartments. Extensor tenosynovitis was more common than flexor(26 v 4 cases). US synovitis 'only' was seen in $76 \%$, US tendon disease 'only' in $7 \%$ and concomitant pathology in $17 \%$. Correlation with inflammatory markers was not seen with only 3 patients presenting with significantly elevated CRP $(>15)$ and ESR $(>20)$. Though CCP was more commonly seen compared to $\operatorname{RhF}(32 \%$ vs $17 \%)$, just over half did not have positive antibodies. Conclusion: Our observational study found that in early RA, mild-to-moderate GSUS and PDUS in the UCJ is the commonest wrist US pathology with tenosynovitis of the $2^{\text {nd }}$ and $4^{\text {th }}$ extensor compartments. Severe disease and erosions were very uncommon. The pattern of these US wrist pathologies are likely to reflect the initial disease course of these patients that present to our EIA service (i.e. early RA patients). Furthermore, biochemical markers do not seem to be useful in these patients and serology may not be present in over $50 \%$. Therefore, clinicians who are running EIA diagnostic services with US should be expectant of milder-to-moderate US findings when diagnosing early RA and not be misled by the absence of more severe findings. Our understanding of US pathologies in the RA disease course therefore needs to be more nuanced and further work in other specific joint areas may help to elaborate what US pathology is most common in early RA.

Disclosure of Interests: None declared

DOI: 10.1136/annrheumdis-2019-eular.932 None declared, Iris Eshed: None declared, Simon Krabbe: None declared, Daniel Glinatsi: None declared, Jakob Møllenbach Møller: None declared, Mikkel Ǿstergaard Grant/research support from: Abbvie, Celgene, Centocor, Merck, Novartis, Consultant for: Abbvie, BMS, Boehringer-Ingelheim, Celgene, Eli Lilly, Hospira, Janssen, Merck, Novartis, Novo, Orion, Pfizer, Regeneron, Roche, and UCB, Speakers bureau: Abbvie, BMS, Boehringer-Ingelheim, Celgene, Eli Lilly, Hospira, Janssen, Merck, Novartis, Novo, Orion, Pfizer, Regeneron, Roche, and UCB

DOI: 10.1136/annrheumdis-2019-eular.1951 
disease is active - making the films useful as a baseline but not much help in getting a timely diagnosis and treatment. Modern imaging techniques, including ultrasound and magnetic resonance imaging (MRI), which can reveal early, non-bony signs of $\mathrm{RA}$ that are invisible on X-ray Objectives: To conduct a comparative analysis of the possibility of MRI in RA patients in the early diagnosis of the disease with the data X-ray of hands and feet

Methods: The study included 56 patients with a reliable diagnosis of RA aged from 17 to 66 years, with disease duration from 4 months to 1 year. Verification of the diagnosis of RA was carried out in accordance with the diagnostic APA criteria (1987). The criteria for the selection of patients was the presence of disease activity. Patients were mainly with the II degree of disease activity. All patients underwent radiography of the small joints of the hands and feet, and 28 patients underwent MRI with dominant hands

Results: The study showed certain changes in the structures of the joint based on MRI in the early stages of RA. So, according to MRI of the brushes, it was revealed: thickening of the synovial membrane - in 23 (86\%) patients; marginal erosion and subchondral cysts - in 21 (75\%) patients; bone erosions - in $25(90 \%)$ patients; phenomena of destruction of the articular cartilage (thinning, ulceration and destruction) - in 16 $(57 \%)$ patients; effusion to the joints - in 19 (68\%) patients and tenosynovitis - in 18 (64\%) patients. In turn, the radiological data of the hands and feet showed that erosive arthritis was found in $23(41 \%)$ patients and marginal erosions - in 22 (39\%) patients

Conclusion: Thus, MRI of the hands plays an important role in the early diagnosis of RA, since it allows visualizing the characteristic changes in all joint structures in RA. MRI is a highly effective diagnostic method in diagnosis of RA, which in combination with other methods of research helps to establish the diagnosis at an early stage diseases and timely appointment of adequate basic treatment unlike radiography

\section{REFERENCES:}

[1] Klarlund M, Ostergaard M, Jensen KE, Madsen JL, Skjodt H, Lorenzen I. Magnetic resonance imaging, radiography, and scintigraphy of the finger joints: one year follow up of patients with early arthritis - the TIRA group. Ann Rheum Dis 2000; 59:521 -528

[2] McQueen FM, Stewart N, Crabbe J, et al. Magnetic resonance imaging of the wrist in early rheumatoid arthritis reveals a high prevalence of erosions at four months after symptom onset. Ann Rheum Dis 1998; 57:350 -356

Disclosure of Interests: None declared

DOI: 10.1136/annrheumdis-2019-eular.2067

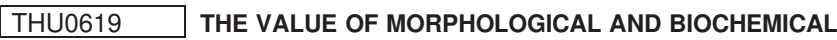 MAGNETIC RESONANCE IMAGING (MRI) OF RADIOCARPAL CARTILAGE FOR THE DIFFERENTIATION OF RHEUMATOID ARTHRITIS AND OSTEOARTHRITIS}

Philipp Sewerin, Lino Sawicki, Christoph Schleich, Daniel Abrar, Matthias Schneider, Stefan Vordenbäumen, Benedikt Ostendorf. Universitätsklinikum Düsseldorf, Düsseldorf, Germany

Background: Using high-field $M R I$ to differ morphologically between $O A$ and RA.

Objectives: To evaluate the value of morphological and biochemical, contrast-agent free high-field ( 3 Tesla) MRI of the radiocarpal cartilage to differ between osteoarthritis (OA) and rheumatoid arthritis (RA).

Methods: The group consisted of 47 subjects, who were examined during the period from October 2016 to December 2017. The clinical dominant hand of 11 patients suffering from early rheumatoid arthritis (RA) (German ArthroMark cohort, $\varnothing$ 52.8years; min: 32; max 74), seropositive (ACPA and/or RF), disease duration < 6 months (range $2-23$ weeks)), 10 with osteoarthritis $(\mathrm{OA})(\varnothing 48.55 \pm 15.23$ years, $\min : 34$ years, max: 68 years, disease duration $\varnothing 6$ years \pm 8.29$)$ and 26 healthy volunteers $(\varnothing$ $46.55 \pm 17.55$ years, min: 20 years, max: 79 years) were examined with a $3 T$ MRI scanner, prospectively. Morphological and biochemical assessment of radiocarpal cartilage was performed using DESS, TrueFISP and T2* images.

Results: Morphological sequences demonstrated significantly higher cartilage damage in RA and OA compared to healthy controls (DESS: $p=$ $0.01, p=0.0004$; TrueFISP: $p=0.02, p=0.0001$ ), while there was no significant difference between RA and OA patients. With biochemical MRI using $\mathrm{T}^{*}$ imaging, patients with $\mathrm{OA}$ showed higher cartilage integrity compared to patients with RA ( $p=0.01)$.
Conclusion: Morphological and biochemical MRI of radiocarpal cartilage could be helpful to differentiate between RA and OA patients. Both, RA and $\mathrm{OA}$, are associated with cartilage damage measured by morphological MRI of the hand. Hence, OA was associated with more loss of cartilage integrity compared to RA using biochemical MRI, whereby only early RA patients were analyzed in this first evaluation. Non-contrast-agent morphological and biochemical MRI could be a non-invasive tool to investigate cartilage integrity in RA and $O A$ patients and could help to differ disease pattern in the future.

Acknowledgement: We would like to thank Erika Rädisch for the assis tance in receiving the MRI scans.

Disclosure of Interests: Philipp Sewerin: None declared, Lino Sawicki: None declared, Christoph Schleich: None declared, Daniel Abrar: None declared, Matthias Schneider Grant/research support from: GlaxoSmithKline and UCB Pharma for performing the LuLa-study., Speakers bureau: Chu gai, Stefan Vordenbäumen: None declared, Benedikt Ostendorf: None declared

DOI: 10.1136/annrheumdis-2019-eular.4831

\section{THU0620 THE PHYSIOLOGICAL FINGER JOINT ARCHITECTURE - AGE-RELATED INCREASE OF EROSIONS AND OSTEOPHYTES IN THE JOINTS OF HEALTHY INDIVIDUALS}

David Simon ${ }^{1}$, Andreas Berlin ${ }^{1}, 2$, Koray Tascilar ${ }^{1}$, Sara Bayat ${ }^{1}$, Klaus Engelke $^{1}$, Jürgen Rech ${ }^{1}$, Camille Figuereido ${ }^{1,3}$, Axel Hueber ${ }^{1,4}$, Georg Schett ${ }^{1}$, Arnd Kleyer ${ }^{1}$. ${ }^{1}$ Friedrich-Alexander University Erlangen-Nuremberg (FAU) and Universitätsklinikum Erlangen, Department of Internal Medicine 3, Rheumatology and Immunology, Erlangen, Germany; ${ }^{2}$ University Hospital Würzburg, Würzburg, Germany, ${ }^{3}$ School of Medicine from São Paulo University (FMUSP)., Sao Paolo, Brazil; ${ }^{4}$ Klinikum Bamberg, Bamberg, Germany

Background: The "normal" finger joint architecture has not yet been defined and could change in the course of life. Therefore the objective was to assess the physiological finger joint architecture of healthy individuals and the relation of structural changes to age and sex.

Objectives: Therefore the objective was to assess the physiological finge joint architecture of healthy individuals and the relation of structura changes to age and sex using high-resolution quantitative computed tomography (HR-pQCT) of the hands.

Methods: Healthy individuals without rheumatic diseases and other comor bidities were recruited through a field campaign and received HR-pQCT examination of the Metacarpophalangeal $2 / 3$ and Proximal Interphalangeal $2 / 3$ joints of one hand. The number of erosions and osteophytes was quantified across different sexes and age decades $(6$ decades within the age range of $21-80$ years). ${ }^{1,2,3}$

Results: 120 healthy individuals (10 women and 10 men in each decade) were recruited. Bone erosions [median (Q1-Q3), 1 (0-2)] and osteophytes [2 (1-4)] were found in both sexes without significant differences. How ever, structural changes increased with age: the overall incidence rate ratio (IRR) for the number of erosions and osteophytes per age were $1.04(95 \% \mathrm{Cl} 1.03-1.06 ; 95 \% \mathrm{Cl} 1.03-1.05)$, which indicates a $4 \%$ increase in the number of erosions and osteophytes per year. The use of the $3^{\text {rd }}$ decade as the reference demonstrated that healthy individuals in the age decades from 50 years had higher IRR for erosion number $\left(6^{\text {th }}, 7^{\text {th }}, 8^{\text {th }}\right.$ decade: $4.87(2.20-11.75), 6.81(3.08-16.46)$ and $6.92(3.11$ $16.79)$ ) compared to younger subjects $\left(4^{\text {th }}, 5^{\text {th }}\right.$ decade: $1.80(0.69-4.87)$, $1.53(0.59-4.10))$. The IRRs of osteophytes also indicate a progressive increase after the fifth decade, with IRRs of 2.32 (1.32-4.17), 4.17 (2.38 $7.49)$ and $6.86(3.97-12.20)$ for the $6^{\text {th }}, 7^{\text {th }}$ and $8^{\text {th }}$ decades, respectively. Conclusion: Structural changes in the finger joints of healthy individuals are age-related. While being rare under 50 years of age, erosions and osteophytes accumulate above the age of 50 , suggesting that the thresh old between "normal" and "pathological" shifts with increasing age.

\section{REFERENCES:}

[1] Simon D, Kleyer A, Stemmler, et al. Age- and Sex-Dependent Changes of Intra-articular Cortical and Trabecular Bone Structure and the Effects of Rheumatoid Arthritis. J Bone Miner Res. 2017 Apr

[2] Figueiredo CP, Simon D, Englbrecht M, et al. Quantification and Impact of Secondary Osteoarthritis in Patients With Anti-Citrullinated Protein Antibody-Positive Rheumatoid Arthritis. Arthritis Rheumatol. 2016 Sep

[3] Figueiredo CP, Kleyer A, Simon D, et al. Methods for segmentation of rheumatoid arthritis bone erosions in high-resolution peripheral quantitative computed tomography (HR-pQCT). Semin Arthritis Rheum. 2018 Apr; 